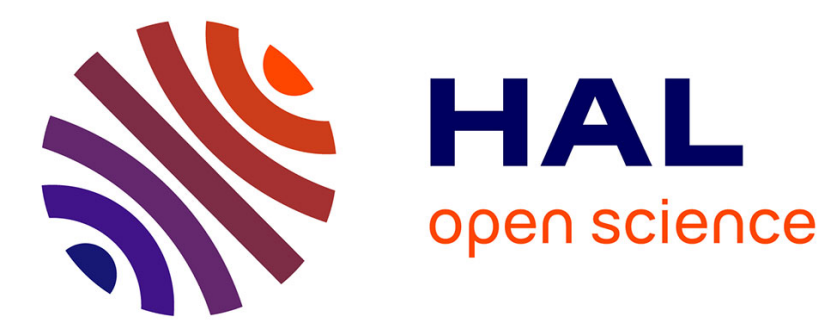

\title{
Design, development, and preliminary evaluation of a highly wearable exoskeleton
}

Monica Malvezzi, Tommaso Lisini Baldi, Alberto Villani, Federico Ciccarese, Domenico Prattichizzo

\section{To cite this version:}

Monica Malvezzi, Tommaso Lisini Baldi, Alberto Villani, Federico Ciccarese, Domenico Prattichizzo. Design, development, and preliminary evaluation of a highly wearable exoskeleton. RO-MAN 2020 29TH IEEE INTERNATIONAL CONFERENCE ON ROBOT AND HUMAN INTERACTIVE COMMUNICATION, Aug 2020, Naples, Italy. hal-02908376

\section{HAL Id: hal-02908376 https://hal.science/hal-02908376}

Submitted on 28 Jul 2020

HAL is a multi-disciplinary open access archive for the deposit and dissemination of scientific research documents, whether they are published or not. The documents may come from teaching and research institutions in France or abroad, or from public or private research centers.
L'archive ouverte pluridisciplinaire HAL, est destinée au dépôt et à la diffusion de documents scientifiques de niveau recherche, publiés ou non, émanant des établissements d'enseignement et de recherche français ou étrangers, des laboratoires publics ou privés. 


\title{
Design, development, and preliminary evaluation of a highly wearable exoskeleton
}

\author{
Monica Malvezzi ${ }^{1}$, Tommaso Lisini Baldi ${ }^{1}$, Alberto Villani ${ }^{1}$, Federico Ciccarese ${ }^{1}$, and Domenico Prattichizzo ${ }^{1,2}$
}

\begin{abstract}
We present the design of a highly wearable exoskeleton of hand fingers that can be used for rehabilitation applications. One of the main challenges in the design of this type of device is to reduce as much as possible the encumbrance and weight, and at the same time to guarantee performance suitable to provide a realistic and reliable motion to the user. In the proposed solution each finger is actuated by means of a single motor, and finger joint rotations are coupled to produce a natural and intuitive movement of the finger, for this reason, we exploited the concept of postural synergies. The paper presents the main design steps, the criteria adopted to choose the mechanical structure of the exoskeleton, and the features of its prototype. Compared to other solutions present in the literature, the device presented in this paper has limited weight ( $40 \mathrm{~g}$ per finger) with an interesting level of performance in terms of force $(15 \mathrm{~N})$.
\end{abstract}

\section{INTRODUCTION}

Interacting with the human body, and in particular with the human hands, is beneficial in several fields such as rehabilitation [1], human-robot interaction [2], and gaming [3]. In these contexts, limited weight, wearability, and intuitiveness of use represent key points since they improve the effectiveness of the process. Wearable devices have the advantages of being portable and well integrated into people habits, providing valuable information to the users. In the next future, such technology will increasingly become more a consistent part of rehabilitation practices and will br more integrated with our clothing and sometimes even part of our bodies [4], [5].

However, most of the existing solutions to reproduce realistic stimuli for haptics and rehabilitation purposes are not completely wearable or portable, usually they rely on structured environments and/or on grounded and bulky hardware.

More recently, the availability of miniaturized actuators and lightweight materials led to the development of extremely wearable devices to return haptic stimuli, typically thimbles, able to generate tactile cues to the user finger pulp. Examples and perspectives of these innovations are described in [6], where the authors overview the challenges

\footnotetext{
${ }^{1}$ Università degli Studi di Siena, Dipartimento di Ingegneria dell'Informazione, Via Roma 56, 53100 Siena, Italy. \{malvezzi,lisini, prattichizzo\}@diism.unisi.it

${ }^{2}$ Istituto Italiano Tecnologia, Advanced Robotics Department, Via Morego 30, 16163 Genoa, Italy

The research leading to these results has received funding from European Union's Horizon 2020 Research and Innovation Programme under Grant Agreement n. 688857 of the project "SoftPro"
}

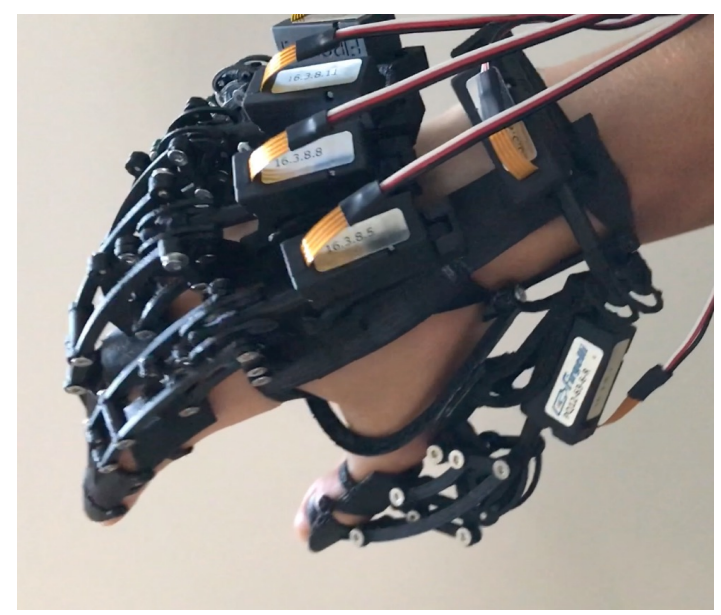

Fig. 1: Hand exoskeleton overview. Each finger is has one linear actuator that moves the proximal and intermediate phalanges. Their motion is coupled with an articulated mechanism reproducing the first postural synergy. Index, middle, ring, and pinky fingers have the same exoskeleton structure, with different size. Thumb exoskeleton has two motors, it has not been described in this paper.

in designing wearable haptic device. In that work, a 3-DOF (Degrees of Freedom) cable-driven wearable tactile device is presented. Other wearable tactile solutions are described in [7] and [8]. The portability and enlargement of the work space that we obtain moving from grounded to wearable solutions comes at the cost of loosing active or passive mechanical support to the patient's rehabilitative activities. In addition, the kinesthetic part of the solicitation is often omitted in extremely wearable devices.

Hand exoskeletons, both in haptics and in rehabilitation scenario, represent the intermediate stage between grounded and fingertip cutaneous devices. They are wearable and have not limitations in the workspace, moreover they can provide mechanical guide and realistic cutaneous and kinesthetic stimuli for hand articulations. In such contexts, the upper limb plays an important role for all daily activities, and various exoskeleton devices have been already developed as support tools for this part of the body. Notwithstanding the technological developments and the evidence of clinical effectiveness of robotic technologies for upper-limb neurorehabilitation, there are still some limits in their diffusion [9]. Technological and economic barriers together with commu- 
nication biases between the producers of the technologies are still open issues.

For what concerns exoskeleton for rehabilitation and training, examples are [10] and [11]. In the former, a hand exoskeleton for accurate force displaying is presented. For each finger, three degrees of freedom are actuated. The latter describes a device based on hand cable-driven approach that involves hand and wrist. In [12] and [13], Iqbal et al. introduce a hand exoskeleton that allows full range of motion and can exert bi-directional forces on the phalanges.

For what concerns the exploitation of exoskeleton in rehabilitation, in [14] the authors present a highly under-actuated exoskeleton for tracking and force feedback. The proposed design implements a novel set of kinematics leading to a transparent mechanism thought for hand rehabilitation. Leonardis et al. in [15] present an EMG-driven robotic hand exoskeleton for bilateral active training of grasp motion in stroke patients. Other interesting exoskeletons for rehabilitation applications are presented in [16], [17], [18], [19]. Interested readers can find a comprehensive review of hand exoskeletons for rehabilitative and assistance applications in [20]. In Table I we report a concise comparison among the proposed and the aforementioned exoskeletons.

One of the main problems (and challenges) in designing hand exoskeleton is that the hand has a complex mechanical structure with many degrees of freedom and constraints. In theory, a fully actuated solution would need one actuator for each degree of freedom. Typically each finger can be represented as a 4-DoF serial kinematic chain, so we would need four actuators for each actuated finger. Actuators are among the elements that have the higher impact in the overall device weight and encumbrance. Thus, to reduce the weight and inertial loads that may fatigue the user, the actuators are positioned on the back of the hand (or wrist) and the motion is transmitted to the actuated joints through proper transmission systems. A reduced number of actuators are already used in CyberGrasp ${ }^{\mathrm{TM}}$ [21] and into the exoskeleton presented in [22]. However, the dimensions of these exoskeleton are such as to reduce the wearability. A one motor exoskeleton is presented in [23]. It is small and lightweight but its mechanical structure allow grasping only in virtual reality applications. Similar examples of light and small exoskeleton are Dexmo [24] and the Rutgers Masters II [25]. The former uses one actuator per finger and bound rigidly the proximal of finger. The latter clutters the hand with palm located linear motors.

In this work, we present the main design steps and a preliminary prototype of a highly wearable hand exoskeleton for rehabilitative applications (Fig. 1), designed to maximize the lightness and portability. The proposed device has only one motor per finger, that actuates both the proximal and intermediate phalanges, and is able to apply bi-directional forces. The mechanical transmission is designed to implement the simplifying concept of postural synergies, introduced by the neuroscientific studies presented in [26]. Such studies demonstrated that notwithstanding the human hand intrinsic complexity, the way humans control it in most of the

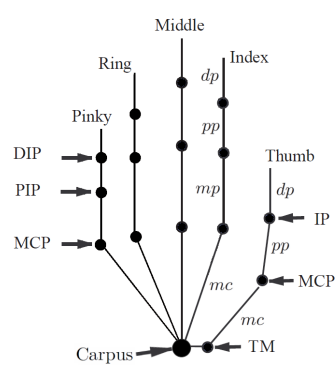

(a)

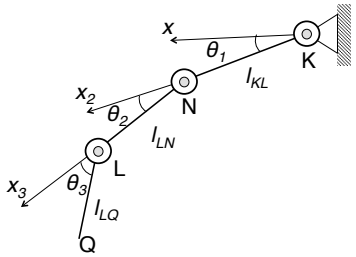

(b)
Fig. 2: Model of the human hand: (a) structure of the hand, joints and links; (b) scheme of the index finger considering only the flexion/extension motion.

everyday life tasks is rather simple and most of the variability of hand postures can be represented by means of a limited number of parameters, the postural synergies. More recently, these concepts were extended to robotics, to design simple and versatile robotic hands [27], [28]. We exploit the concept of postural synergies in the design of a mechanical system that couples hand joints and therefore limits the number of actuators to one per finger. To the best of our knowledge, this represents one of the first attempts to develop and validate a wearable finger exoskeleton based on postural synergies.

\section{FINGER MECHANICAL MODEL}

The human hand has a complex mechanical structure that can be represented as a mechanism composed of five serial kinematic chains, the fingers, sharing a common base on the wrist. A complete human hand model has about 30 DoFs [29], but for the sake of simplicity and without loss of generality, we use a simplified kinematic hand structure. We modeled each finger as a kinematic chain, with one universal joints (two intersecting, orthogonal revolute joints) and two hinges (see Fig. 2a). In accordance with [30], we modeled the metacarpophalangeal (MCP) joint of the index, middle, ring and pinky fingers as a 2-DoF universal joint (one for adduction/abduction and another for flexion/extension). The proximal interphalangeal (PIP) and distal interphalangeal (DIP) joints have one DoF each. If the hand is not affected by particular pathologies, we can assume that the rotation axes of PIP, DIP and the flexion/extension one of the MCP are parallel. In this paper we focus on index finger flexion/extension motion, but it is worth pointing out that the same model, with different phalanx sizes, can be replicated for the middle, ring and pinky.

The human skill of manipulating objects strongly depends on the thumb kinematics and on its capability of opposing the other fingers. In human hands the thumb has at least 5 DoFs: 2 DoFs in trapeziometacarpal (TM) joint, 2 DoFs in metacarpophalangeal (MCP) joint, and 1 DoF in interphalangeal (IP) joint. The analysis of the thumb is not reported here for the sake of space and will be included in an extended version of this work.

considering only the flexion/extension motion, the scheme 


\begin{tabular}{|c|c|c|c|c|c|c|c|}
\hline Name & HEXOTRAC & BRAVO & HANDEXOS & IntelliArm & EMG PoliMi & HES & UNISI \\
\hline Reference & [14] & [15] & {$[16]$} & [17] & {$[18]$} & [19] & - \\
\hline Number of fingers & 5 & 5 & 5 & 5 & 5 & 4 & 4 \\
\hline Maximum Grasping Force & $\sim 12 \mathrm{~N}$ & $30 \mathrm{~N}$ & $10 \mathrm{~N}$ & Not Provided & $100 \mathrm{~N}$ & Not Provided & $\sim 15 \mathrm{~N}$ \\
\hline Number of actuated DoFs & 1 & 1 per finger & 3 per finger & 1 & 2 & 1 per finger & 1 per finger \\
\hline $\begin{array}{l}\text { Method of force } \\
\text { measurement }\end{array}$ & $\begin{array}{l}\text { Strain gauges } \\
\text { and load cells }\end{array}$ & $\begin{array}{l}\text { Proportional } \\
\text { voltage- } \\
\text { torque } \\
\text { transfer } \\
\text { function }\end{array}$ & $\begin{array}{c}\text { Magnetic } \\
\text { encoders, } \\
\text { piezoresis- } \\
\text { tive, and Hall } \\
\text { sensors }\end{array}$ & Torque sensor & $\begin{array}{l}\text { Potentiome- } \\
\text { ters }\end{array}$ & $\begin{array}{c}\text { Embedded in } \\
\text { the } \\
\text { servomotors }\end{array}$ & $\begin{array}{c}\text { Not } \\
\text { Applicable }\end{array}$ \\
\hline $\begin{array}{l}\text { Hand orientation } \\
\text { measurement }\end{array}$ & Yes & No & No & Yes & No & No & No \\
\hline $\begin{array}{l}\text { Implemented safety } \\
\text { measures }\end{array}$ & Yes & No & No & No & No & No & Yes \\
\hline Actuator control loop rate & $1 \mathrm{kHz}$ & $25 \mathrm{~Hz}$ & $100 \mathrm{~Hz}$ & Not Provided & Not Provided & Not Provided & $500 \mathrm{~Hz}$ \\
\hline $\begin{array}{l}\text { Type and form factor of } \\
\text { Electronics }\end{array}$ & $\begin{array}{l}\text { Embedded } \\
\text { microcon- } \\
\text { troller }\end{array}$ & $\begin{array}{l}\text { Embedded } \\
\text { microcon- } \\
\text { troller }\end{array}$ & $\begin{array}{l}\text { External } \\
\text { controller }\end{array}$ & $\begin{array}{l}\text { External } \\
\text { controller }\end{array}$ & $\begin{array}{l}\text { External } \\
\text { controller }\end{array}$ & $\begin{array}{l}\text { Embedded } \\
\text { microcon- } \\
\text { troller }\end{array}$ & $\begin{array}{l}\text { Embedded } \\
\text { Microcon- } \\
\text { troller }\end{array}$ \\
\hline Power supply & $18 \mathrm{~V}, 250 \mathrm{~W}$ & Not Provided & Not Provided & Not Provied & Not Provied & $\begin{array}{l}8 \text { Ni-Mh AA } \\
\text { Batteries }\end{array}$ & $12 \mathrm{~V}, 15 \mathrm{~W}$ \\
\hline $\begin{array}{l}\text { Require a host system for } \\
\text { operation }\end{array}$ & Yes & Yes & Yes & Yes & Yes & No & Yes \\
\hline
\end{tabular}

TABLE I: Comparison between the proposed exoskeleton (indicated as UNISI, last column) and other solutions available in the literature.

of the index, middle, ring and little fingers is approximately the same and is depicted in Fig. 2b. Points $K, N$ and $L$ represent the axes of MCP, PIP, and DIP joints, respectively. Point $Q$ represents the fingertip. Furthermore, we indicate with $\theta_{1}, \theta_{2}$, and $\theta_{3}$ their relative rotation angles. We denote with $l_{K N}, l_{L N}$, and $l_{N Q}$ the lengths of the links representing the proximal, intermediate and distal phalanges, respectively.

For a given set of angles $\boldsymbol{\theta}=\left[\theta_{1}, \theta_{2}, \theta_{3}\right]^{\mathrm{T}}$ the evaluation of fingertip coordinates $\mathbf{p}_{Q}=\left[x_{Q}, y_{Q}\right]^{\mathrm{T}}$, i.e. the solution of the forward kinematics problem, is straightforward. By differentiating the forward kinematics relationships with respect to time we can easily express the velocity of the fingertip $\mathbf{v}_{P}$ as a function of joint angular velocities $\dot{\boldsymbol{\theta}}$ :

$$
\mathbf{v}_{Q}=\mathbf{J}_{i} \dot{\boldsymbol{\theta}}
$$

where $\mathbf{J}_{i} \in \mathbb{R}^{3 \times 2}$ is the matrix representing the index Jacobian. In a static equilibrium condition, the transpose of $\mathbf{J}_{i}$ relates the force applied to the fingertip $\mathbf{f}_{Q}$ to the torques $\boldsymbol{\tau}=\left[\tau_{1}, \tau_{2}, \tau_{3}\right]^{\mathrm{T}}$ applied to joints:

$$
\boldsymbol{\tau}+\mathbf{J}_{i}^{\mathrm{T}} \mathbf{f}_{Q}=0 .
$$

Hand synergies: The aforementioned studies in neuroscience [26] showed that, even if the human hand has a complex mechanical structure, with many degrees of freedom, a set of variables with a limited dimension is able to represent a wide range of hand poses in everyday life tasks. More recently, these concepts were brought to robotics [27], [28] and inspired the development of a new type of underactuated robotic hands. In this paper we exploit the results of our previous studies on synergies [28] to set the reduction ratio between $\theta_{2}$ and $\theta_{1}$, in particular, the value of this ratio obtained in the first postural synergy was adopted. In this way, even if joint rotations are coupled, we expect that this constraint appears intuitive and natural to the user. Even if the exoskeleton is designed to replicate a single hand motion (first synergy), we decided to have a motor per finger because designing a transmission system able to take into account the un-actuated adduction abduction motions, while guaranteeing a bi-directional force, would lead to a more complex mechanical structure.

\section{FINGER EXOSKELETON STRUCTURE}

\section{A. Overall structure}

A sketch of the exoskeleton kinematic structure for the index finger is shown in Fig. 3. Three main parts can been identified, each part is composed of some components, assumed as rigid bodies, connected by joints. Components sketched in black are fixed on the hand, in particular, component 1 is fixed on the back of the palm, componentt 6 is attached on the proximal phalanx, 10 is on the intermediate one, and 11 is on the distal one. Components 10 and 11 are connected with a revolute joint resembling DIP articulation, such a rotation is not constrained by the exoskeleton. The geometry of these component is designed according to wearable and ergonomic considerations: these component are in contact with the human hand and have to be sufficiently light and comfortable for the user, so they have to be adapted to specific hand characteristics. Among all the specific features that we need to set when designing them, we can broadly distinguish between parameters necessary to 


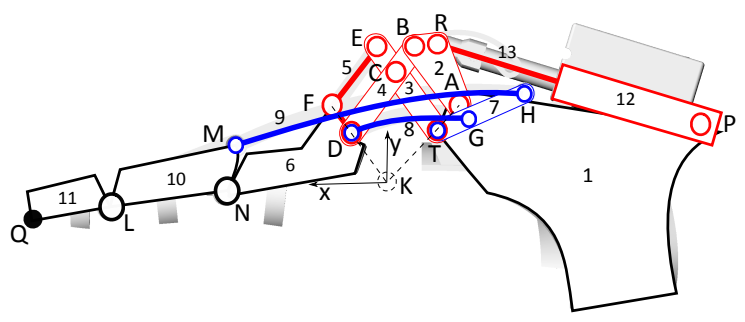

Fig. 3: Scheme of the kinematic structure exoskeleton for the index finger. Small circles represent hinge (revolute) joints, while thick lines and shapes represent system components. Different colors are used to indicate the main parts of the structure: black components are connected to user's hand, red components realize the RCM and the connection with the actuator, blue components realise the coupling between PIP and DIP rotations.

adapt the elements to the hand, and parameters that influence exoskeleton kinematics.

In this paper, we focus mainly in this last set of parameters. Considering the components sketched in black in the figure, the only variables that influence exoskeleton kinematics are the lengths $l_{K N}$, that represents the distance between MCP and PIP joint axes and is necessary to define the proper dimensions of all the mechanism elements, $l_{L N}$ and $l_{L Q}$, necessary to evaluate the trajectories of $L$ and $Q$ points. These parameters are user-specific and are the inputs for exoskeleton kinematic design.

The components depicted in red realise the actuation and the remote center of rotation: components 12 and 13, represent the mobile parts of a linear actuator, while components $2,3,4$, and 5 realise the remote center of rotation in $K$, where the MCP axis is supposed to pass.

Finally, the components sketched in blue implement the kinematic coupling between the MCP and the PIP according to a ratio defined on the basis of the first postural synergy. In what following we detail the analysis of each part. Table II summarizes the main geometrical parameters, their variability ranges and the values adopted for the index finger prototype described in this paper.

\section{B. Actuating the MCP joint, the Remote Center of Motion}

A common problem that is featured when designing a hand exoskeleton is that it is not possible to directly actuate the MCP revolute joint. This restriction comes from the physical impossibility to reproduce in the mechanical device the corresponding revolute joint.

The problem has been solved by exploiting the concept of Remote Center of Motion (RCM), a quite comprehensive review of mechanisms realising a RCM is provided in [31]. In our application, we choose a simple and common solution, composed of two articulated parallelograms connected to form a six-bar mechanism. A mechanism similar to the one described in this paper was adopted for example in [10], [15].

Its scheme is reported in red in Fig. 3. Points $T$ and $A$ are the axes of revolute joints connecting the first parallelogram

\begin{tabular}{|l|l|l||l|l|l|}
\hline Variable & Value, unit & Range & Variable & Value,unit & Range \\
\hline \hline$l_{K N}$ & $38 \mathrm{~mm}$ & {$[30-45]$} & $x_{P}$ & $67.4 \mathrm{~mm}$ & - \\
\hline$l_{L N}$ & $31,9 \mathrm{~mm}$ & {$[25-35]$} & $y_{P}$ & $22.4 \mathrm{~mm}$ & - \\
\hline$l_{L Q}$ & $20.0 \mathrm{~mm}$ & {$[15-25]$} & $l_{G T}$ & $8.45 \mathrm{~mm}$ & {$[5-15]$} \\
\hline$l_{A B}$ & $20 \mathrm{~mm}$ & {$[15-30]$} & $l_{G H}$ & $16.3 \mathrm{~mm}$ & {$[10-25]$} \\
\hline$l_{B C}$ & $9 \mathrm{~mm}$ & {$[5-15]$} & $l_{G D}$ & $30.9 \mathrm{~mm}$ & {$[25-40]$} \\
\hline$l_{B R}$ & $0 \mathrm{~mm}$ & {$[010]$} & $l_{M H}$ & $77.3 \mathrm{~mm}$ & {$[60-80]$} \\
\hline$l_{A R}$ & $19.7 \mathrm{~mm}$ & $l_{A B}+[5-15]$ & $l_{M N}$ & $13 \mathrm{~mm}$ & {$[12-18]$} \\
\hline
\end{tabular}

TABLE II: Exoskeleton main geometrical parameters, range of variability and values adopted in the paper.

(components 2,3,4) to the hand palm 1, points $F$ and $D$ connects the second parallelogram (components 3,4,5) to the link 6 , connected to the proximal phalanx. The parallelograms share the links 3 and 4 .

In such a mechanism, the motion of the link 6 is a pure rotation with respect to point $K$, intersection between the lines through $\overline{F D}$ and $\overline{T A}$. If the device is designed so that the point $K$ corresponds to the axis of the MCP joint, the proximal phalanx can be actuated by rotating the link 2 . The rotation is achieved in the prototype by means of a linear actuator whose mobile part is connected to point $R$ and whose fixed end is connected to the exoskeleton fixed part in point $P$ trough two revolute joints. Using this type of actuation the exoskeleton can apply bi-directional forces. In other terms it can constrain both flexion and extension motions of the finger.

These components of the mechanism were dimensioned taking into account heterogeneous and even conflicting criteria. Increasing the dimension, i.e. lengthening parallelogram edges $l_{A B}$ and $l_{B C}$ has the advantage of augmenting the torque that can be applied to hand articulation for a given actuator force and at the same time leave more free space for the MCP joint, but, on the other hand, increases overall encumbrance and weight of the exoskeleton. For the index finger of a hand with an average dimension, we adopted $l_{A B}=20 \mathrm{~mm}$ and $l_{B C}=9 \mathrm{~mm}$ [32], obtaining an acceptable trade-off between the above mentioned criteria.

\section{Actuating the PIP joint, joint coupling}

The proposed exoskeleton uses a single motor to actuate both the MCP and the PIP joints. The rotations of such joints are mechanically coupled using a double four-bar mechanism, sketched in blue in Fig. 3. The motion of link 9 is constrained by link 8 , connected in $D$ to the proximal phalanx 6 . The link 7 constrains the motion of the middle phalanx 10 with respect to the proximal one (6). A straightforward direct kinematic analysis allows to evaluate the coupling between MCP and PIP rotation constrained by exoskeleton structure. In general, since the forward kinematic relationship is non-linear, such coupling is not a fixed ratio and may vary. In this realisation we are interested in keeping such a ratio as much constant as possible and to set its value to that obtained by considering the first postural synergy. In particular, for the index finger, results described in [26] and 
available in the repository [33], set $\frac{\theta_{2}}{\theta_{1}}=2.27$.

We evaluated $L$ point trajectory by assuming $l_{K N}=38 \mathrm{~mm}$ and $l_{L N}=31.9 \mathrm{~mm}$, imposing the first synergy coupling and varying $\theta_{1}$ in the range $[0.08,0.8] \mathrm{rad}$, corresponding to a open-close movement of the hand. We divided the rotation angle in $N_{s}=73$ steps with an amplitude of $\Delta \theta_{1}=0.01$ rad. Let us indicate with $\mathbf{p}_{L, s y n, i}=\left[x_{L, s y n, i}, y_{L, s y n, i}\right]^{\mathrm{T}}$ the obtained coordinates of point $L$ with respect to a reference frame with origin in $K$ and oriented as indicated in Fig. 3, corresponding to the sample $\theta_{1, i}$, with $i=1 \cdots N_{s}$.

Standard forward kinematic analysis allows to evaluate the $L$ coordinates of the same point according to the constraints imposed by exoskeleton kinematics, being $\mathbf{p}_{L, \text { exo }, i}=$ $\left[x_{L, \text { exo }, i}, y_{L, \text { exo }, i}\right]^{\mathrm{T}}$. In this case the trajectory will depend on mechanism dimension, let us collect in vector 1 the geometric variables on which the coupling mechanism depends, namely $\mathbf{l}=\left[l_{G D}, l_{G H}, l_{O G}, l_{M N}, l_{M H}\right]$. We indicate with $e(\mathbf{l})$ a measure of the error between the synergy and the exoskeleton trajectories of point $L$, that we defined as

$$
e(\mathbf{l})=\sum_{i=1}^{N_{s}}\left(\mathbf{p}_{L, \text { exo }, i}-\mathbf{p}_{L, \text { syn }, i}\right)^{2} \text {. }
$$

Thus, we attempted to solve the problem

$$
\min _{\mathbf{l}}(e(\mathbf{l})), \mathbf{l} \in \mathcal{L},
$$

where $\mathcal{L}$ is a set of possible values of 1 , defined to obtain a mechanically feasible and wearable solution.

We obtained $l_{G D}=30.89 \mathrm{~mm}, l_{G H}=16.26 \mathrm{~mm}, l_{G T}=$ $8.45 \mathrm{~mm}, l_{M N}=13.0, l_{M H}=77.3 \mathrm{~mm}$. Fig. 4 shows the trajectories for points $N$, and $L$ corresponding to PIP and DIP axis, respectively, compared with the trajectories of the same points imposed by synergy constraint. Fig. 5 sketches the main points of the exoskeleton in the first and last configurations and highlights the trajectories of points $N$ and $L$.

\section{Unactuated joints}

The MCP adduction/abduction motion is not actuated in the current implementation of the exoskeleton, but will be one of the topics of its further developments. In this prototype such motion is constrained, since the actuator is fixed on the hand palm. However, the mechanical design has been developed so that with a simple modification of the fixed support we can unlock the rotation of the actuated unit with respect to the hand palm support, over an axis corresponding to MCP adduction/abduction rotation.

The DIP joint is not actuated as well, for two main reasons. Firstly its actuation would require a more complex and therefore less wearable design. Secondly, since on the fingertip the tactile part of the haptic stimuli is more relevant with respect to the kinesthetic one, we planned to integrate such device with tactile thimbles described in [34], [8].

\section{EXOSKELETON VELOCITY AND FORCE ANALYSIS}

The kinematic analysis can be completed with velocity and acceleration evaluation for all the meaningful points of

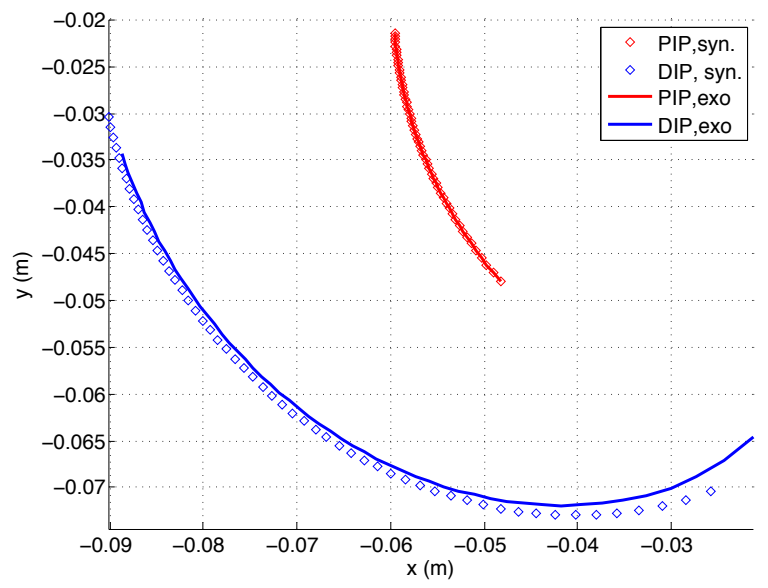

Fig. 4: Kinematic simulation: trajectories of points $L$ (red, corresponding to the PIP joint) and $N$ (blue, corresponding to the DIP joint) for different values of $\theta_{1}$ angle, varying from 0.08 to $0.8 \mathrm{rad}$. Continuous lines: trajectories constrained by the exoskeleton, dotted lines: trajectories corresponding to the first postural synergy defined in [26].

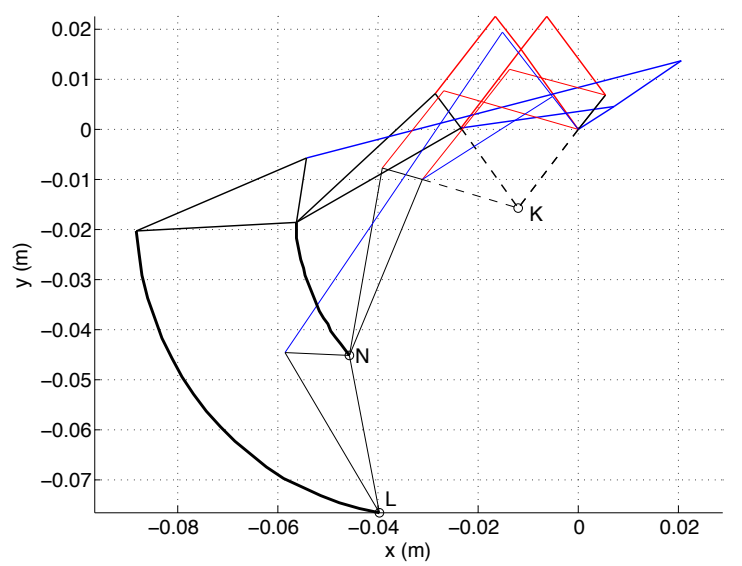

Fig. 5: Kinematic simulation: trajectories of points $L$ (corresponding to the PIP joint) and $N$ (corresponding to the DIP joint) for different values of $\theta_{1}$ angle, varying from 0.08 to $0.8 \mathrm{rad}$.

the exoskeleton. In this case the specification of one input velocity is required: since the exoskeleton is actuated by a linear actuator (elements 12 and 13 in Fig. 3), we can assume its linear velocity $\dot{s}$ as input. The velocity analysis can be straightforwardly carried out, it consists in solving a linear system of equations, whose coefficients depends on exoskeleton configuration. We are in particular interested in finding the relationship between hand actuated joints angular velocities $\dot{\theta}_{1}$ and $\dot{\theta}_{2}$, and $\dot{s}$. This relationship is linear and can be expressed as

$$
\dot{\boldsymbol{\theta}}=\mathbf{J}_{e} \dot{s}
$$

where $\mathbf{J}_{e}$ has dimensions $2 \times 1$, it represents exoskeleton Jacobian and its elements in general depends on exoskeleton configuration. Recalling Eq. (1) and assuming a fixed ratio 


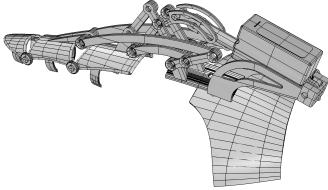

(a) (b)

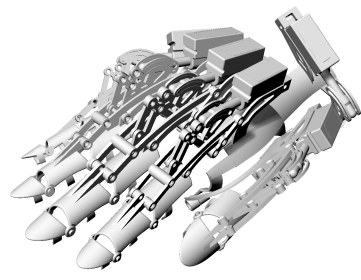

Fig. 6: 3D model of the finger exoskeleton. a) index finger (b) whole hand, thumb actuation is not described in this paper and will be the focus of future works.

between DIP and PIP rotations $\frac{\theta_{3}}{\theta_{2}}$, we can evaluate hand fingertip velocity $\mathbf{v}_{Q}$ as a function of $\omega$ :

$$
\mathbf{v}_{Q}=\mathbf{J}_{t} \dot{s}
$$

where $\mathbf{J}_{t}=\mathbf{J}_{h} \tilde{\mathbf{J}}_{e}$, and $\tilde{\mathbf{J}}_{e}$ represents the exoskeleton Jacobian extended to include DIP rotation.

The transpose of exoskeleton Jacobian relates the torques applied by the exoskeleton to hand joints $\tau=\left[\tau_{1}, \tau_{2}\right]^{\mathrm{T}}$ to the force $f_{a}$ applied by the actuator to the link 2 , specifically

$$
f_{a}=\mathbf{J}_{e}^{\mathrm{T}} \boldsymbol{\tau}
$$

and, through Eq. (2) we can express the relationship between a force to be rendered on the fingertip and the actuator force $f_{a}$ :

$$
f_{a}=\mathbf{J}_{e}^{\mathrm{T}} \mathbf{J}_{i}^{\mathrm{T}} \mathbf{f}_{Q}
$$

\section{EXOSKELETON DESIGN AND PROTOTYPE}

The mechanism realising exoskeleton actuation was designed considering the kinematics factors described in the previous section and the specific biomechanical properties of the human hand. In particular, to have a correct and comfortable transmission, the elements of the mechanism have to be designed so that the distance between points $K$ and $N$ is as close as possible to proximal phalanx length. Fig. 6 shows an overview of the overall device. Fig. 6a shows a 3D CAD of the actuation system for the index finger, while Fig. $6 \mathrm{~b}$ depicts the extension to the whole hand.

For the device actuation, in the prototype presented in this paper we adopted an Actuonix PQ12 (Actuonix Motion Device Inc., CA) linear actuator, positioned on the exoskeleton part connected to hand palm and constrained to apply a pulling/pushing force directly to link indicated as 2 in Fig. 3. The connection between actuator and link 2 , i.e., point $R$ position, could be manually regulated by sliding actuator mobile end along a circular guide and fixing it in the desired position. As discussed in Sec. III, this feature allows to change force/velocity ratios and therefore to set their values according to the application. The actuator that we choose for the prototype is a R-series, whose main mechanical characteristics are summarised in Table III. As it can be seen, the actuator can exert a maximum pulling force up to $45 \mathrm{~N}$ and can be controlled in position as a servo motor. A simple position control with selectable proportional gain

\begin{tabular}{|l|l|l|}
\hline Feature & Units & Value \\
\hline Peak Power Point & & $30 \mathrm{~N} 8 \mathrm{~mm} / \mathrm{s}$ \\
\hline Peak Efficiency Point & & $12 \mathrm{~N} 12 \mathrm{~mm} / \mathrm{s}$ \\
\hline Maximum speed (no load) & $\mathrm{mm} / \mathrm{s}$ & 15 \\
\hline Maximum Force & $\mathrm{N}$ & 45 \\
\hline Stroke & $\mathrm{mm}$ & 20 \\
\hline Input Voltage & $\mathrm{VDC}$ & 6 \\
\hline Mass & $\mathrm{g}$ & 15 \\
\hline Mechanical backlash & $\mathrm{mm}$ & 0.25 \\
\hline Audible noise & $\mathrm{dB} 45 \mathrm{~cm}$ & 55 \\
\hline
\end{tabular}

TABLE III: Actuonix linear actuator PQ12-R main specifications.

was implemented in the prototype by means of an AtMega based microcontroller. Varying the proportional gain of the position control it was possible to realize some preliminary simulations of interactions with materials with different stiffness properties. The power supply is provided by a $7.4 \mathrm{~V}$ LiPo battery. Both the microcontroller and the battery pack are in a wearable bracelet. The total weight of the armband is $90 \mathrm{~g}$. Taking into account the kinematic analysis described in Sec. IV and the characteristics of the actuator, we evaluated the maximum force at the fingertip and its maximum velocity. Such values depends on the dimensions of exoskeleton links and on exoskeleton configuration. The results obtained for the index finger, with the dimensions introduced in Sec. III and summarized in Table II, is shown in Fig. 7.

The structure of the exoskeleton was realized in ABS through an additive manufacturing process. Its total weight including the actuator is about $40 \mathrm{~g}$ per finger. More in detail, each motor weighs $15 \mathrm{~g}$, while the ABS structure contributes with $25 \mathrm{~g}$ The geometry of exoskeleton elements was verified by means of a structural analysis taking into account the maximum effort that can be generated by the actuator. Some images of the prototype are shown in Fig. 8. The device is easy to wear, and its dimensions and weight are limited. Fig. 8a and $8 \mathrm{~b}$ show two reference configurations, open and closed, while Fig. 8c and 8d show how the exoskeleton, when not actuated, does not significantly affect human hand mobility and the user can perform tasks as writing or grasping objects while wearing the device.

\section{ExOSKELETON EVALUATION}

As introduced in Sect. I, the proposed exoskeleton exploits the concept of postural synergy for controlling the joint motion, thus the finger trajectory. In [26] the authors investigated the postural synergies hypothesis by recording a large data set of grasping poses from subjects that were asked to mime grasps of a set of 57 objects. A Principal Components Analysis (PCA) of this data reported that more than $80 \%$ of the variance could be accounted with the first two principal components. In other words, instead of controlling the single 20 DoFs of a human hand, one or two joints coupling leading to coordinated motions of the hand could be used to achieve 

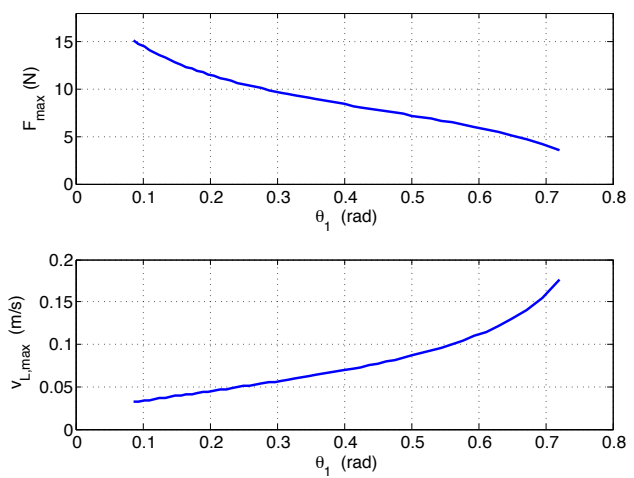

Fig. 7: Maximum equivalent force and maximum equivalent velocity of the index fingertip as a function of the MCP rotation $\theta_{1}$, evaluated according to mechanism analysis and actuator performance.

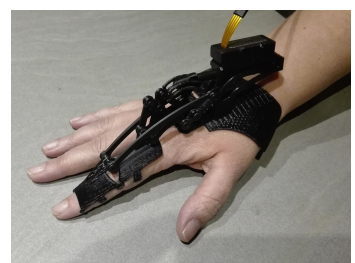

(a)

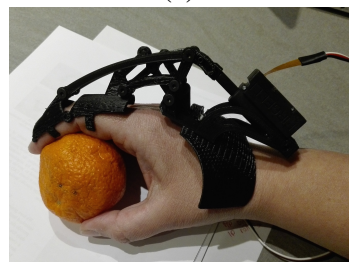

(c)

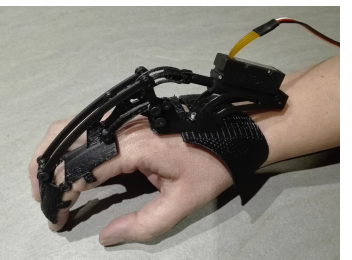

(b)

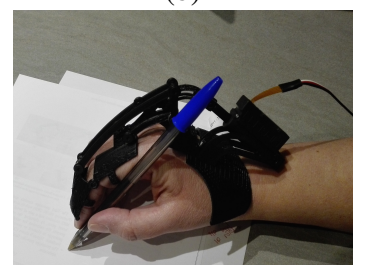

(d)
Fig. 8: Wearing the exoskeleton prototype: a) open configuration, (b) close configuration, (c) while grasping an object, and (d) while writing.

many of the grasps used in everyday life. In this work we mechanically implemented a mechanism to recreate motions belonging to the first synergy (first PC). To validate our approach we decide to compare simulated and predicted data with real hand motions in grasping objects.

A Vicon optical tracking system consisting of ten cameras was used to provide reference measurements of the user's hand. Ten healthy subjects were involved in the validation procedure (age range 25-51, eight males and two females). None of the participants reported any deficiencies in the perception abilities (including vision, hearing, touch, and proprioception). Each user was asked to wear a glove equipped with 9 passive retroreflective markers. Only the index finger was tracked by the system. We put two markers in each phalanx and three markers in the palm. Then, participants were asked to freely open and close the hand, and grasp one of the objects placed in a table in front of them. Objects are from a standard set for manipulation research [35]. Raw data were post-processed using Vicon Nexus software to extract

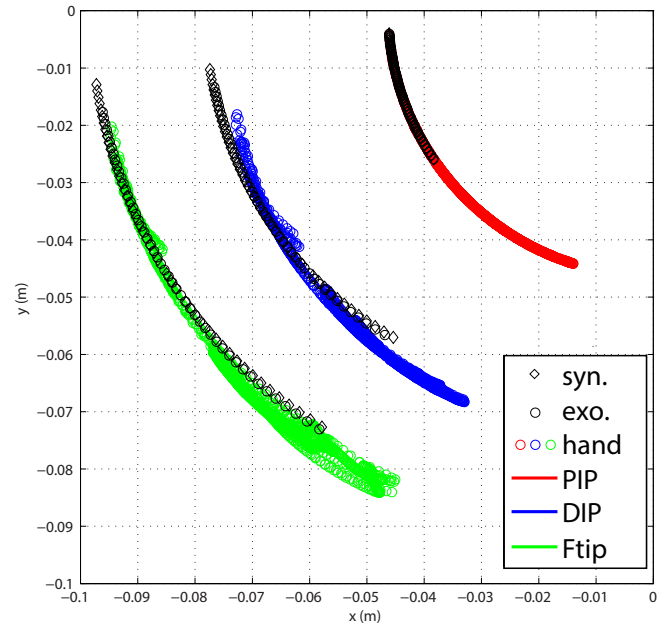

Fig. 9: Representative trajectories comparison: in red trajectories of points $L$ (corresponding to the PIP joint position) in blue $N$ (corresponding to the DIP joint position), and in green $Q$ (corresponding to the fingertip position) for different values of $\phi$ angle. Black circle points: trajectories constrained by the exoskeleton, Black diamond points: trajectories corresponding to the first postural synergy [26], and colored pints: real human trajectory acquired using the optical tracking system, without any constraint.

position of the markers and the joint values. In Fig. 9 real hand trajectories are compared with the exoskeleton and first postural synergy ones.

\section{CONCLUSION}

This work introduces a wearable exoskeleton for hand finger actuation, suitable for haptic application in rehabilitation treatments. As discussed in the initial part of the manuscript, the design of this type of device is challenging since it requires the matching between several contrasting requirements. Ideally, the exoskeleton should be very light, should not over constrain the hand, should apply forces and torques suitable for accurately reproducing desired haptic simulations. The paper proposes a solution in which each finger is actuated with only one motor. The exoskeleton considers the flexion/extension motion only and its structure is designed to apply a torque to the MCP and PIP joints. First postural synergy has been exploited to realize the coupling between PIP and MCP joints, and a RCM mechanism was adopted for the actuation of the MCP joint. The prototype shows a good wearability, lightness, and versatility. Finger movements constrained by the exoskeleton were compared with free hand movements measured with a tracking system: the obtained motions are similar, preliminary confirming that the exoskeleton motion can be perceived as natural by the user.

Future developments of this study will include the extension to the whole hand and in particular to the thumb finger, whose mechanical structure is more complex, the possibility of unconstraining the adduction/abduction motion 
of the fingers, the research of more efficient and reliable actuation systems, a more detailed analysis of exoskeleton elements stress and strain, an optimization of the design and an experimental testing in real rehabilitation scenarios.

\section{REFERENCES}

[1] H. Zhou and H. Hu, "Human motion tracking for rehabilitation-a survey," Biomedical Signal Processing and Control, vol. 3, no. 1, pp. 1-18, 2008.

[2] M. A. Goodrich, A. C. Schultz et al., "Human-robot interaction: a survey," Foundations and Trends $®$ in Human-Computer Interaction, vol. 1, no. 3, pp. 203-275, 2008.

[3] A. Bleiweiss, D. Eshar, G. Kutliroff, A. Lerner, Y. Oshrat, and Y. Yanai, "Enhanced interactive gaming by blending full-body tracking and gesture animation," in ACM SIGGRAPH ASIA 2010 Sketches. ACM, 2010, p. 34.

[4] L. Meli, D. Barcelli, T. L. Baldi, and D. Prattichizzo, "Hand in air tapping: A wearable input technology to type wireless," in 2017 26th IEEE International Symposium on Robot and Human Interactive Communication (RO-MAN). IEEE, 2017, pp. 936-941.

[5] T. Lisini Baldi, S. Scheggi, L. Meli, M. Mohammadi, and D. Prattichizzo, "GESTO: A Glove for Enhanced Sensing and Touching Based on Inertial and Magnetic Sensors for Hand Tracking and $\mathrm{Cu}-$ taneous Feedback," IEEE Transactions on Human-Machine Systems, vol. 47, no. 6, pp. 1066-1076, Dec 2017.

[6] C. Pacchierotti, S. Sinclair, M. Solazzi, A. Frisoli, V. Hayward, and D. Prattichizzo, "Wearable haptic systems for the fingertip and the hand: taxonomy, review, and perspectives," IEEE transactions on haptics, vol. 10, no. 4, pp. 580-600, 2017.

[7] D. Leonardis, M. Solazzi, I. Bortone, and A. Frisoli, "A wearable fingertip haptic device with 3 dof asymmetric 3-rsr kinematics," in World Haptics Conference (WHC), 2015 IEEE. IEEE, 2015, pp. 388-393.

[8] F. Chinello, M. Malvezzi, C. Pacchierotti, and D. Prattichizzo, "Design and development of a 3rrs wearable fingertip cutaneous device," in Advanced Intelligent Mechatronics (AIM), 2015 IEEE International Conference on. IEEE, 2015, pp. 293-298.

[9] G. Turchetti, N. Vitiello, S. Romiti, E. Geisler, and S. Micera, "Why effectiveness of robot-mediated neurorehabilitation does not necessarily influence its adoption," IEEE Reviews in Biomedical Engineering, vol. 7, pp. 143-153, 2014.

[10] M. Fontana, A. Dettori, F. Salsedo, and M. Bergamasco, "Mechanical design of a novel hand exoskeleton for accurate force displaying," in Robotics and Automation, 2009. ICRA'09. IEEE International Conference on. IEEE, 2009, pp. 1704-1709.

[11] M. Dragusanu, T. Lisini Baldi, Z. Iqbal, D. Prattichizzo, and M. Malvezzi, "Design, development and control of a tendon-actuated exoskeleton for wrist rehabilitation and training," in Proc. IEEE Int. Conf. on Robotics and Automation, Paris, FR, June 2020.

[12] J. Iqbal, N. G. Tsagarakis, and D. G. Caldwell, "Design of a wearable direct-driven optimized hand exoskeleton device," in Proc. 4th Int. Conf. Advances in Computer-Human Interactions. Citeseer, 2011, pp. $142-146$.

[13] J. Iqbal, N. Tsagarakis, and D. Caldwell, "Human hand compatible underactuated exoskeleton robotic system," Electronics Letters, vol. 50, no. 7, pp. 494-496, 2014.

[14] I. Sarakoglou, A. Brygo, D. Mazzanti, N. G. Hernandez, D. G. Caldwell, and N. G. Tsagarakis, "Hexotrac: A highly under-actuated hand exoskeleton for finger tracking and force feedback," in 2016 IEEE/RSJ International Conference on Intelligent Robots and Systems (IROS). IEEE, 2016, pp. 1033-1040.

[15] D. Leonardis, M. Barsotti, C. Loconsole, M. Solazzi, M. Troncossi, C. Mazzotti, V. P. Castelli, C. Procopio, G. Lamola, C. Chisari et al., "An emg-controlled robotic hand exoskeleton for bilateral rehabilitation," IEEE transactions on haptics, vol. 8, no. 2, pp. 140$151,2015$.

[16] A. Chiri, N. Vitiello, F. Giovacchini, S. Roccella, F. Vecchi, and M. C. Carrozza, "Mechatronic design and characterization of the index finger module of a hand exoskeleton for post-stroke rehabilitation," IEEE/ASmE Transactions on mechatronics, vol. 17, no. 5, pp. 884894, 2012.
[17] Y. Ren, H.-S. Park, and L.-Q. Zhang, "Developing a whole-arm exoskeleton robot with hand opening and closing mechanism for upper limb stroke rehabilitation," in Rehabilitation Robotics, 2009. ICORR 2009. IEEE International Conference on. IEEE, 2009, pp. 761-765.

[18] M. Mulas, M. Folgheraiter, and G. Gini, "An emg-controlled exoskeleton for hand rehabilitation," in Rehabilitation Robotics, 2005. ICORR 2005. 9th International Conference on. IEEE, 2005, pp. 371-374.

[19] R. Conti, B. Allotta, E. Meli, and A. Ridolfi, "Development, design and validation of an assistive device for hand disabilities based on an innovative mechanism," Robotica, pp. 1-15, 2015.

[20] P. Heo, G. M. Gu, S.-j. Lee, K. Rhee, and J. Kim, "Current hand exoskeleton technologies for rehabilitation and assistive engineering," International Journal of Precision Engineering and Manufacturing, vol. 13, no. 5, pp. 807-824, 2012.

[21] CyberGlove Systems Inc. The CyberGrasp system. [Online]. Available: http://www.cyberglovesystems.com/cybergrasp

[22] Z. Ma and P. Ben-Tzvi, "Design and optimization of a five-finger haptic glove mechanism," Journal of Mechanisms and Robotics, 03 2015.

[23] I. Choi, E. Hawkes, D. Christensen, C. Ploch, and S. Follmer, "Wolverine: A wearable haptic interface for grasping in virtual reality," 10 2016, pp. 986-993.

[24] X. Gu, Y. Zhang, W. Sun, Y. Bian, D. Zhou, and P. Kristensson, "Dexmo: An inexpensive and lightweight mechanical exoskeleton for motion capture and force feedback in vr," 05 2016, pp. 1991-1995.

[25] M. Bouzit, G. Burdea, G. Popescu, and R. Boian, "The rutgers master ii-new design force-feedback glove," Mechatronics, IEEE/ASME Transactions on, vol. 7, pp. 256 - 263, 072002.

[26] M. Santello, M. Flanders, and J. F. Soechting, "Postural hand synergies for tool use," Journal of Neuroscience, vol. 18, no. 23, pp. $10105-$ $10115,1998$.

[27] A. Bicchi, M. Gabiccini, and M. Santello, "Modelling natural and artificial hands with synergies," Phil. Trans. R. Soc. B, vol. 366, no. 1581, pp. 3153-3161, 2011.

[28] D. Prattichizzo, M. Malvezzi, M. Gabiccini, and A. Bicchi, "On motion and force controllability of precision grasps with hands actuated by soft synergies," IEEE transactions on robotics, vol. 29, no. 6, pp. 1440-1456, 2013.

[29] J. Lee and T. L. Kunii, "Model-based analysis of hand posture," IEEE Computer Graphics and applications, vol. 15, no. 5, pp. 77-86, 1995.

[30] S. Mulatto, A. Formaglio, M. Malvezzi, and D. Prattichizzo, "Using postural synergies to animate a low-dimensional hand avatar in haptic simulation," IEEE transactions on haptics, vol. 6, no. 1, pp. 106-116, 2013.

[31] G. Zong, X. Pei, J. Yu, and S. Bi, "Classification and type synthesis of 1-dof remote center of motion mechanisms," Mechanism and Machine Theory, vol. 43, no. 12, pp. 1585-1595, 2008.

[32] F. Tosi, Ergonomia e progetto. F. Angeli, 2006.

[33] M. Bianchi and M. V. Liarokapis, "Handcorpus, a new open-access repository for sharing experimental data and results on human and artificial hands," in IEEE World Haptics Conference (WHC), 2013.

[34] D. Prattichizzo, F. Chinello, C. Pacchierotti, and M. Malvezzi, "Towards wearability in fingertip haptics: a 3-dof wearable device for cutaneous force feedback," IEEE Transactions on Haptics, vol. 6, no. 4, pp. 506-516, 2013.

[35] B. Calli, A. Singh, A. Walsman, S. Srinivasa, P. Abbeel, and A. M. Dollar, "The ycb object and model set: Towards common benchmarks for manipulation research," in 2015 International Conference on Advanced Robotics (ICAR), July 2015, pp. 510-517. 